\title{
Ocular surface regeneration with autologous biomaterials
}

\begin{abstract}
Purpose: To report a mini review on novel biomaterials applied for ocular surface regeneration.

Methods: A systematic review of the literature.

Results: Human-derived amniotic membrane is the most preferred biomaterial to regenerate ocular surface because of its ease of access and low immunogenicity. Whereas, biological instability, lack of optimized preparation protocols, and risk of transmission of infection are still major issues that make its use controversial. Human blood-derived biomaterials have gained popularity to reconstruct and regenerate the ocular surface with a really basic principle and even low cost. Platelet-rich plasma (PRP) and platelet-rich-fibrin (PRF) are the two types of platelet enriched biomaterials that have been using in various clinical fields to improve wound healing via their excellent chemotactic and physical properties. Autologous biomaterials obtained from blood can be used in order to regenerate ocular surface.
\end{abstract}

Conclusion: This review explores critical needs in ocular surface problems where conventional therapies are not well suited and the current demand to overcome corneal blindness.

Keywords: ocular surface regeneration, human-derived amniotic membrane, autologous biomaterials, human blood-derived biomaterials, platelet-rich fibrin
Volume 8 Issue I - 2018

Gamze Dereli Can

Department of Ophthalmology, Bursa Yuksek Ihtisas Training and

Research Hospital, Turkey

Correspondence: Gamze Dereli Can, Department of Ophthalmology, Bursa Yuksek Ihtisas Training and Research Hospital, Bursa, Turkey, Tel +905555807l40,

Email dereli_gmz@hotmail.com

Received: February II, 20I8 | Published: February 26, 2018

\section{Introduction}

The "Ocular Surface System" includes the specialized epithelium of the cornea and conjunctiva, main and accessory lacrimal glands, meibomian glands, and eyelashes which are in an excellent relationship to maintenance of smooth and wet characters of the anterior window of the eye ball. Since the primary function of the system components is to provide and protect the transparency of the cornea, the corneal epithelium may attributable the chief element in the system. ${ }^{1}$ Because of the continuity of the epithelial barrier of the ocular surface with no breaks, a physician should consider to regenerate the whole surface of the eye with a unique and broad-spectrum treatment strategy. Various ocular and systemic diseases such as dry eye syndrome, blepharitis associated with meibomian gland dysfunction, ocular allergies, surface scarring from topical treatments, chemical injuries, thermal burns, and immunological disorders (e.g. Stevens Johnson syndrome and mucous membrane pemphigoid) can severely disturb the ocular surface integrity that may result in blurry vision, redness, pain, and even blindness and decrease in the quality of life. ${ }^{2-4}$ After definite diagnosis, care and management strategies generally demand highly specialized expertise. Although most of the diseases of the ocular surface can be treated with medical strategies, some advanced grade disorders can only be improved with surgical treatments that required some supportive surgical equipments and graft materials.

\section{Discussion}

Successful ocular surface reconstruction includes to restore neuroanatomic integration of the surface epithelium and stem cell stromal niche at the limbal region. Therefore, there are two main steps to achieve the best results for ocular surface regeneration. Firstly, urgent surface reconstruction with various biomaterials obtained from different sources, and secondly to proceed the grafted surface integrity with the support of stem cell therapies. ${ }^{5,6}$ There are several natural biomaterials applied as a surgical graft material such as humanderived amniotic membrane (HAM),${ }^{3}$ conjunctiva, ${ }^{7}$ autologous or allogenic lamellar/full thickness corneal grafts $^{8}$ and scleral patches,${ }^{9}$ autologous fascia lata, ${ }^{10}$ and cadaveric or animal-derived pericardium ${ }^{11}$ or dura mater ${ }^{12}$ with varying success rates. However, HAM is the most preferred one because of its ease of access and low immunogenicity. The HAM promotes epithelialization by acting as a basement membrane and releases growth factors such as epidermal growth factor and keratocyte growth factor which are important for the wound healing. Further, it has anti-inflammatory and anti-scarring effects by the inhibition of transforming growth factor beta signalling in the regeneration process. However, the preparation of HAM is complicated and even expensive. In addition, a strong tissue banking expertise is crucial to prevent inadvertent complications. The risk of transmission of some serious pathogens such as hepatitis-B and human immune deficiency virus always exist and cannot be excluded even in the presence of very strict procedures. Furthermore, HAM is a natural but an allogenic matrix, and immunologic response to allografts is the major concern even if it is low. ${ }^{13}$

Recently, human blood-derived biomaterials have gained popularity to reconstruct and regenerate the ocular surface with a really basic principle and even low cost. Platelet-rich plasma (PRP) is a first-generation platelet concentrate which was discovered in 
1970s. ${ }^{14}$ As a consequence of its high amount of platelet, it releases several growth factors such as vascular endothelial growth factor, transforming growth factor beta, epithelial growth factor, plateletderived growth factor, and matrix proteins as vitronectin, fibronectin, and thrombospondin-1which are located in the alpha granules of platelets. ${ }^{15}$ The preparation process comprises consecutive two centrifugation steps with individual's own blood sample and chemicals such as anti-coagulant, calcified thrombin and calcium chloride. ${ }^{16}$ The anti-coagulant is essential to avoid the self-coagulation process and calcified thrombin and calcium chloride should be used for the activation of platelet degranulation. However, the aforementioned chemicals are generally animal-derived which may have an impact on the safety of the application. The PRP has been applied in oral, maxillofacial, orthopedic, cardiovascular, plastic and reconstructive specialties, as well as eye diseases (E-PRP) such as dry eye, corneal epithelial defects and ulcers, and ocular surface regeneration after corneal perforations. ${ }^{17,18}$ More recently, platelet-rich fibrin (PRF) was introduced as a second-generation platelet concentrate with its superior features. In order to prepare the PRF; a ten-mL blood sample is withdrawn from a peripheral vein of the individual in a glass tube without anticoagulant.

The blood sample is then centrifuged at $2.700 \mathrm{rpm}$ (approximately $400 \mathrm{~g}$ ) for $12 \mathrm{~min}$. After centrifugation, the final sample consists of 3 distinct layers. A fibrin clot is obtained in the middle of the tube between the acellular plasma layer at the top and red corpuscles at the bottom. This fibrin clot is collected from the tube by using forceps. Because of the three-dimensional fibrin architecture and sustained release of growth factors during fibrin degradation make this innovative material very suitable for tissue engineering applications. ${ }^{19}$ Moreover, the one-step preparation protocol does not include animalderived materials and harmful chemicals such as anti-coagulant or thrombin. The Choukroun's PRF was first used in ocular surface regeneration by Can et al. ${ }^{13}$ in an animal study. Can emphasized that autologous PRF membrane has excellent mechanical and chemical features and can be used as a growth factor-enriched endogenous scaffold for ocular surface reconstruction. Another study by Can et al. ${ }^{20}$ showed that the temporary PRF membrane grafting may be an alternative intervention to avoid impending corneal perforation in cases with severe descemetocele.

\section{Conclusion}

This review explores critical needs in ocular surface problems where conventional therapies are not well suited and novel strategies are reasonably required. The PRP, PRF and their modifications can be applied as a safe auto-origin graft material for ocular surface regeneration when the conventional methods are not sufficient.

\section{Conflicts of interest}

None.

\section{Acknowledgments}

None.

\section{References}

1. Gipson IK. The ocular surface: the challenge to enable and protect vision: the Friedenwald lecture. Invest Ophthalmol Vis Sci. 2007;48(10):43914398 .
2. Stern ME, Beuerman RW, Fox RI, et al. The pathology of dry eye: the interaction between the ocular surface and lacrimal glands. Cornea. 1998;17(6):584-589.

3. Shimazaki J, Yang HY, Tsubota K. Amniotic membrane transplantation for ocular surface reconstruction in patients with chemical and thermal burns. Ophthalmology. 1997;104(12):2068-2076.

4. Tsubota K1, Satake Y, Ohyama M, et al. Surgical reconstruction of the ocular surface in advanced ocular cicatricial pemphigoid and StevensJohnson syndrome. Am J Ophthalmol. 1996;122(1):38-52.

5. Azuara-Blanco A, Pillai CT, Dua HS. Amniotic membrane transplantation for ocular surface reconstruction. B J Ophthalmol. 1999;83(4):399-402.

6. Kenyon KR, Tseng SC. Limbal autograft transplantation for ocular surface disorders. Ophthalmology. 1989; 96(5):709-723.

7. Thoft RA. Conjunctival transplantation. Arch Ophthalmol. 1977;95(8):1425-1427.

8. Nishida K1, Yamato M, Hayashida Y, et al. Functional bioengineered corneal epithelial sheet grafts from corneal stem cells expanded ex vivo on a temperature-responsive cell culture surface. Transplantation. 2004;77(3):379-385.

9. Oh JH, Kim JC. Repair of scleromalacia using preserved scleral graft with amniotic membrane transplantation. Cornea. 2003;22(4):288-293.

10. Torchia RT, Dunn RE, Pease PJ. Pease, Fascia Lata Grafting in Scleromalacia Perforans: With Lamellar Corneal-Scleral Dissection. Am J Ophthalmol. 1968;66(4):705-709.

11. Weissgold DJ, Millay RH, Bochow TA. Rescue of exposed scleral buckles with cadaveric pericardial patch grafts. Ophthalmology. 2001;108(4):753758 .

12. Enzenauer RW, Enzenauer RJ, Reddy VB, et al. Treatment of scleromalacia perforans with dura mater grafting. Ophthalmic Surg. 1992;23(12):829 832.

13. Can ME, Çakmak HB, Dereli Can G, et al. A Novel Technique for Conjunctivoplasty in a Rabbit Model: Platelet-Rich Fibrin Membrane Grafting, Journal of ophthalmology. 2016;(2016).

14. Marx RE, Carlson ER, Eichstaedt RM, et al. Platelet-rich plasma: growth factor enhancement for bone grafts. Oral Surgery, Oral Med Oral Pathol Oral Radiol Endod. 1998;85(6):638-646.

15. Marx RE. Platelet-rich plasma (PRP): what is PRP and what is not PRP?, Implant Dent. 2001;10(4):225-228.

16. Wu PI, Diaz R, Borg-Stein J. Platelet-rich plasma. Phys Med Rehabil Clin. 2016;27(4):825-853.

17. Carlson NE, Roach RB. Platelet-rich plasma: clinical applications in dentistry. J Am Dent Assoc. 2002;133(10):1383-1386.

18. Alio JL, Rodriguez AE, WróbelDudzińska D. Eye platelet-rich plasma in the treatment of ocular surface disorders. Curr Opin Ophthalmol. $2015 ; 26(4): 325-332$.

19. Dohan DM, Choukroun J, Diss A, et al. Platelet-rich fibrin (PRF): a second-generation platelet concentrate. Part I: technological concepts and evolution. Oral Surg Oral Med Oral Pathol Oral Radiol Endod. 2006;101(3):e37-e44.

20. Can ME, Dereli Can G, Cagil N, et al. Urgent therapeutic grafting of plateletrich fibrin membrane in descemetocele. Cornea . 2016;35(9):1245-1249. 